\title{
LAS PAUSAS SONORAS Y LOS ALARGAMIENTOS EN ESPAÑOL: UN ESTUDIO PRELIMINAR
}

\author{
FILLED PAUSES AND LENGTHENING IN SPANISH: A PRELIMINARY STUDY
}

\author{
María J. MACHUCA, Joaquim LlisterRI, Antonio Ríos \\ Universitat Autònoma de Barcelona
}

\section{RESUMEN:}

Aunque los fenómenos de hesitación en el discurso - pausas sonoras y alargamientos- se han abordado desde diferentes puntos de vista, son escasos los estudios que analizan sus manifestaciones acústicas en español. En este trabajo se presenta una caracterización preliminar de las hesitaciones que, generalmente, suelen aparecer en el habla espontánea con diferentes funciones discursivas. Se han analizado 180 minutos de conversaciones en las que han participado seis hablantes, con una duración aproximada de 30 minutos cada una, y 30 fragmentos de producción espontánea de un minuto de duración correspondientes a tres sesiones de grabación de 10 locutores. En las primeras se da una interacción entre los participantes, mientras que en las segundas, el hablante sabe que no será interrumpido durante su intervención. El análisis acústico se ha complementado con un estudio perceptivo en el que los oyentes debían valorar la naturalidad y la espontaneidad de los mismos fragmentos presentados con o sin alargamiento y con o sin pausa sonora. Los resultados muestran, en primer lugar, que la pausa sonora más frecuente en español es [e:]. Los valores medios y las desviaciones correspondientes a la duración de los alargamientos y de las pausas sonoras no arrojan diferencias significativas entre estas hesitaciones. No obstante, el rango de los valores medios debería considerarse en la caracterización de tales elementos. Además, los resultados indican que el hecho de que el hablante sepa que no va a ser interrumpido conlleva un incremento de la aparición de hesitaciones en habla espontánea. Los enunciados con pausas y alargamientos se perciben con el mismo grado de naturalidad si se comparan con una versión de los mismos enunciados en la que se han eliminado las hesitaciones, pero no con idéntico nivel de espontaneidad. Finalmente, cabe resaltar que los alargamientos suelen percibirse en el discurso como si el hablante hubiera realizado un silencio.

PALABRAS CLAVE: Hesitaciones, pausas sonoras, alargamientos.

\section{ABSTRACT:}

Hesitations in spontaneous speech -filled pauses and lengthenings- have been approached from different perspectives, but their acoustic characteristics in Spanish have not yet been systematically analyzed. The aim of this preliminary study is to characterize hesitations that are used in spontaneous speech with different discourse functions. The corpus consisted of six spontaneous conversations between 2 participants, with a duration of about 30 minutes each, and 30 spontaneous fragments of a minute of duration corresponding to three sessions recorded by 10 speakers. In the conversations, participants interacted with each other, whereas in the spontaneous fragments, the speaker knew that he would not be interrupted. The acoustic 
analysis has been complemented with a perceptual study in which the listeners assessed the naturalness and the spontaneity of the same fragments presented with or without lengthening and with or without filled pauses. First of all, results show that the filled pause realized as [e:] is the most frequent one in Spanish. There are no significant differences between the duration of lengthenings and the duration of filled pauses; however, the range of temporal values should be taken into account in the characterization of hesitations. Results also reveal that if the speaker knows that he will not be interrupted, the number of hesitation phenomena increases. Fragments with and without hesitations are perceived as having the same degree of naturalness, but not the same level of spontaneity. Finally, it is interesting to note that in spontaneous speech lengthenings might be perceived as empty pauses.

KEY WORDS: Hesitations, fillers, filled pauses, lengthenings. 


\section{INTRODUCCIÓN}

Muchísimos trabajos han abordado el estudio de los fenómenos propios del habla espontánea desde diferentes puntos de vista. Además, en ellos se han empleado distintos términos para hacer referencia a los elementos sonoros -denominados, en ocasiones, de relleno (por el término empleado en inglés, fillers) - que aparecen en el discurso oral.

Los analistas de la conversación se plantean como un objetivo fundamental descubrir de qué manera los turnos de palabra se articulan como la base organizativa de las interacciones verbales. Según Calsamiglia y Tusón (2007), las vocalizaciones, definidas como sonidos o ruidos emitidos por los hablantes, que no son palabras y que se producen en combinación con gestos faciales o de otras partes del cuerpo, desempeñan funciones comunicativas importantes. No obstante, para referirse a las vocalizaciones, otros autores distinguen entre aquellos elementos que están lexicalizados y aquellos que no lo están (Stenström, 1994). Por otra parte, Rose (1998) establece una sistematización de los fenómenos que pueden aparecer en una conversación: falsos comienzos, repeticiones, autocorrecciones, alargamientos y pausas. Entre estas últimas, distingue varios tipos de pausa, que pueden presentarse antes o después de actos de habla, de oraciones o de cláusulas, siempre en posiciones gramaticalmente significativas. Según este autor, pueden ser tanto silenciosas como sonoras, pero son numerosos los investigadores que aluden a las pausas sonoras como los fenómenos empleados más frecuentemente en la conversación (Nicholson, Eberhard y Scheutz, 2010; Wennerstrom, 1994). En Cesteros (2000) se analizan los fenómenos que señalan los cambios de turno en la conversación. Según esta autora, los movimientos tonales marcan de forma directa la zona de transición de esos cambios de turno, mientras que los alargamientos finales y las pausas constituyen elementos secundarios que operan reforzando o cambiando la indicación del momento apropiado para la transición.

En el ámbito de las tecnologías del habla, este tipo de fenómenos ha sido estudiado con el fin de mejorar los sistemas de reconocimiento, y se han descrito como disfluencias o eventos acústicos (Torres, 2006). Rodríguez, Torres y Varona (2001) analizaron un total de 227 diálogos y obtuvieron, sin incluir los alargamientos, un mayor número de casos de pausas sonoras (1028) que de silenciosas (753). Además, Carlson, Gustafson y Strangert (2006) estudiaron qué rasgos prosódicos contribuían a una mejor percepción de estos fenómenos en el habla espontánea para considerarlos en los sistemas de diálogo; estos autores llegan a la conclusión de que el incremento de la duración es la estrategia que contribuye en mayor grado a la percepción de las disfluencias en el habla espontánea. La caída de la frecuencia fundamental $\left(f_{0}\right)$ o la presencia de voz rota (creaky voice) sirven para reforzar perceptivamente aquellos casos en los que la duración es corta. Candeias, Celorico, Proença, Veiga y Perdigão (2013) han creado para el portugués una base de datos para el desarrollo de las tecnologías del habla (HESITA). Estos autores destacan que la frecuencia de aparición de pausas sonoras y de alargamientos es determinante para diferenciar los estilos de habla: 7.34 casos por minuto en habla espontánea frente a 0.22 en lectura. Así mismo, en 
Albalá et al. (2008) se ha observado que la duración de las pausas sonoras y la de las silenciosas mostraban un comportamiento distinto de los hablantes en los dos estilos de habla analizados, lectura y habla espontánea.

Las pausas sonoras y los alargamientos también han sido estudiados en el ámbito clínico como indicadores de la producción oral de los enfermos de Alzheimer. Gayraud, Barkat-Defradas y Lee (2011) observan diferencias significativas entre hablantes sin patologías y pacientes con Alzheimer en la frecuencia de aparición de estos fenómenos, pero no en la duración. Por otra parte, su uso aumenta en relación con el grado de Alzheimer que sufra el paciente (Rodríguez, 2013).

También se ha recurrido al estudio de las pausas sonoras en la adquisición de lenguas extranjeras para explicar el concepto de fluidez en el habla. El trabajo de Sánchez (2002), entre otros, muestra cómo el hablante nativo, frente a los aprendices, es el que emite un mayor número de palabras por minuto y realiza un menor número de pausas sonoras; no obstante, el autor concluye, a partir de una prueba perceptiva, que no existe relación entre la cantidad de pausas y la percepción de una mayor fluidez. Por otro lado, Rose (2013) muestra que los elementos sonoros de relleno que aparecen en la conversación en una segunda lengua dependen de los elementos que utiliza el hablante en su lengua materna.

En ámbitos judiciales se han analizado estos fenómenos para la identificación del locutor (Brander, 2014). Künzel (1997) indicó que las pausas sonoras eran un parámetro consistente para identificar a los hablantes, ya que estos suelen tener preferencia por alguno de los sonidos consonánticos o vocálicos que se pueden alargar en una determinada lengua. Los trabajos de Cicres $(2007,2014)$ son también un ejemplo de este enfoque. No obstante, este autor considera que, si bien las pausas sonoras son útiles para la identificación de los hablantes, en ningún caso un dictamen pericial debe basarse exclusivamente en este análisis, sino que tiene que fundamentarse en la suma de un conjunto de comparaciones lingüísticas.

Algunos autores han señalado que el sonido que se emplea para emitir una pausa sonora depende del sistema fónico de cada lengua, ya que los hablantes producen sonidos que no requieren amplios movimientos articulatorios (Patzold y Simpson, 1995; Stepanova, 2007) o recurren a una vocal no marcada (Gil, 2007). Concretamente, en español puede alargarse cualquier fonema vocálico, excepto la vocal $/ \mathrm{u} /$, aunque la vocal que se alarga más frecuentemente es /e/ (Rebollo, 1997). Entre las consonantes, las que se alargan son /1/, /n/ y /m/. Según Rebollo (1997), los fonemas /e/ y /m/ son los únicos que pueden constituir verdaderas pausas sonoras, ya que pueden insertarse en el discurso sin que necesariamente deriven del alargamiento de una palabra. El hablante utiliza, sobre todo, el alargamiento de los segmentos en posición final para organizar el discurso; este alargamiento acaba con una pausa silenciosa la mayor parte de las veces. El término que emplearemos en el presente trabajo para dar cuenta de los fenómenos mencionados se recoge en el Diccionario de la lengua española (DRAE, 2014), hesitaciones, palabra procedente del latín, que en el corpus CORPES XXI aparece en diferentes obras de autores hispanoamericanos.

El objetivo de esta investigación es proporcionar datos sobre las pausas sonoras y los alargamientos en español considerando una situación comunicativa espontánea. 
Además, se contempla también el hecho de que exista o no interacción en las situaciones comunicativas analizadas. Para ello, se llevan a cabo dos experimentos: un análisis acústico para caracterizar la duración de los elementos sonoros de relleno en el habla espontánea, y un análisis perceptivo para determinar cómo los participantes en una prueba de percepción evalúan estas hesitaciones dentro de un estímulo de habla.

\section{EsTUDIO ACÚSTICO}

\subsection{Metodología}

El corpus empleado en el análisis acústico se ha extraído de diferentes muestras de habla espontánea pertenecientes a corpus grabados para otros proyectos de investigación. En concreto, el corpus de conversaciones espontáneas en las que se produce una interacción entre los dos hablantes forma parte del recogido para el proyecto El español en contacto con el catalán: variación diatópica y bilingüismo (Ministerio de Economía y Competitividad: FFI2012 - 33499). De este corpus, se han seleccionado seis hablantes, tres mujeres y tres hombres. En la selección se ha considerado el grado de dominancia lingüística, eligiendo a hablantes cuya lengua dominante fuera el español; se ha tenido también en cuenta que existiera una relación de amistad entre los dos interlocutores -el investigador y el hablante estudiado- que participan en la conversación, de modo que el intercambio comunicativo se realizara con la mayor espontaneidad posible. Las grabaciones duraban aproximadamente unos 30 minutos, pero, considerando que el sujeto que se analizaba participaba en la conversación tanto como el investigador, la duración del fragmento analizado era de unos 15 minutos.

El resto del corpus corresponde al fragmento de 1 minuto de habla espontánea de 10 hablantes masculinos seleccionados al azar del corpus utilizado para el proyecto VILE, Estudio acústico de la variación inter e intralocutor en español (BFF2001-2551, 2001-2004 y HUM2005-06980, 2005-2008), que, a su vez, era una parte del corpus AHUMADA (Ortega, González y Marrero, 2000). A estos sujetos se les pedía que hablaran durante un minuto sobre cualquier tema que les motivara y sabían que durante ese minuto no iban a ser interrumpidos. Cada uno de estos hablantes fue grabado en tres sesiones separadas en el tiempo; por tanto, en este caso, se dispone de tres minutos para cada locutor.

En total se han analizado 120 minutos de habla espontánea: 90 minutos de conversación con interacción (6 hablantes $\times 15$ minutos) y 30 minutos de lo que podríamos denominar monólogo, sin interacción ( 3 sesiones $\times 1$ minuto $\times 10$ hablantes). En estas grabaciones se han estudiado las pausas sonoras propiamente dichas -es decir, aquellas vocalizaciones o murmullos sonoros que no pertenecen a ninguna categoría léxica y que pueden tener una función comunicativa en el discurso- y los alargamientos -sonidos que forman parte de palabras con una categoría léxica determinada y que pueden tener, al igual que las pausas sonoras, una función comunicativa-. Consideramos como un fenómeno diferente lo que sucedía con la conjunción $y$, ya que 
en el proceso de segmentación se observó que tal elemento estaba a caballo entre la pausa sonora y el alargamiento: había perdido en el discurso su función como categoría léxica, motivo por el que se ha clasificado como un elemento expletivo (véase Cortés, 1991). La tabla 1 muestra el total de casos para cada categoría analizada.

\begin{tabular}{|l|l|l|l|}
\hline & Con interacción & Sin interacción & Total \\
\hline Pausa sonoras & 73 & 83 & 156 \\
\hline Alargamientos & 123 & 158 & 281 \\
\hline Elementos expletivos & 22 & 41 & 63 \\
\hline Total & 218 & 282 & 500 \\
\hline
\end{tabular}

Tabla 1. Número de hesitaciones analizadas

No se han tenido en cuenta aquellos alargamientos que servían para mostrar un énfasis o los que formaban parte de una enumeración, puesto que no se trata de elementos de relleno. Con el objetivo de caracterizar las hesitaciones se han tenido en cuenta las siguientes variables: a) el contexto anterior y siguiente del fenómeno analizado, es decir, si había un fragmento de habla o una pausa silenciosa, b) si aparecen otros elementos de relleno, como chasquidos y palabras comodines, y c) si se detectaba una presencia de voz rota. En los alargamientos, se ha considerado, además, la categoría gramatical de la palabra que se alargaba; y en las pausas sonoras, el elemento vocálico que se utilizaba para emitirlas. En todos los casos se ha analizado la duración del fenómeno.

\subsection{Resultados}

Los resultados que se muestran en la figura 1 ponen de manifiesto que los fenómenos analizados en el habla espontánea siempre aparecen en un porcentaje mayor - calculado sobre el total de los 500 casos analizados- cuando el hablante sabe que no va a ser interrumpido en su discurso (Sin interacción) que cuando puede ser interrumpido en cualquier momento por su interlocutor (Con interacción). No obstante, el valor de significación de una prueba de chi2, en la que la variable independiente es el fenómeno analizado y la dependiente la situación comunicativa, indica que globalmente no hay diferencias significativas $(p=.27)$ en cuanto a la frecuencia de aparición de las hesitaciones en los dos tipos de interacción considerados. 


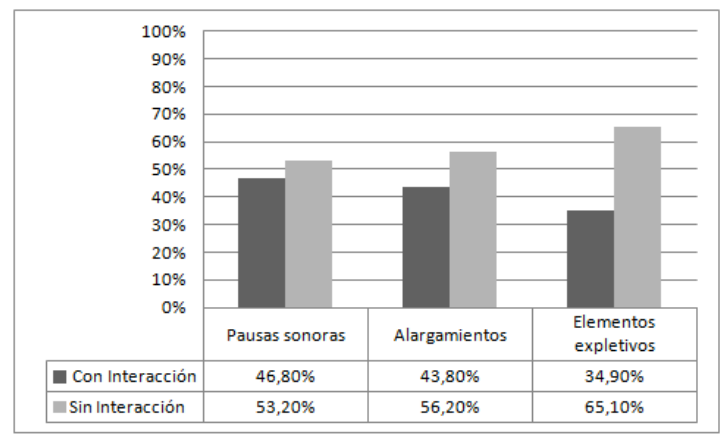

Figura 1. Frecuencia de aparición (en porcentajes) de las hesitaciones en función de la interacción entre los hablantes

Si se considera qué fenómeno aparece en el discurso antes y después de las hesitaciones analizadas (véase la figura 2) se puede afirmar que existen muy pocos casos en los que los alargamientos vienen precedidos de pausas silenciosas (10\%); generalmente, el hablante está produciendo su discurso y emite un alargamiento de una palabra determinada $(88,6 \%$ de los casos). Después de ese alargamiento, o bien se puede encontrar un silencio (un 37,4\% de los casos) o bien el hablante continúa con el discurso (un $56 \%$ de los casos). En cambio, la mayoría de los casos de pausa sonora aparecen en el discurso tanto precedidos de silencio (un $59 \%$ ) como seguidos de habla (un 73,1\%). Hay solo un $31 \%$ de los casos en que el hablante estaba emitiendo el discurso oral y produce una pausa sonora, y menos casos aún $(19,2 \%)$ en los que el hablante realiza una pausa silenciosa después de una pausa sonora. Finalmente, cuando el hablante utiliza y como un elemento expletivo y no como una conjunción, antes suele producir una pausa silenciosa (un 82,5 \% de los casos) y después suele mostrar un comportamiento similar al que ocurre con la pausa sonora. La categoría «Otros», que se muestra en la figura 2, hace referencia a repeticiones o a falsos inicios que pueden encontrarse antes o después de los elementos mencionados.
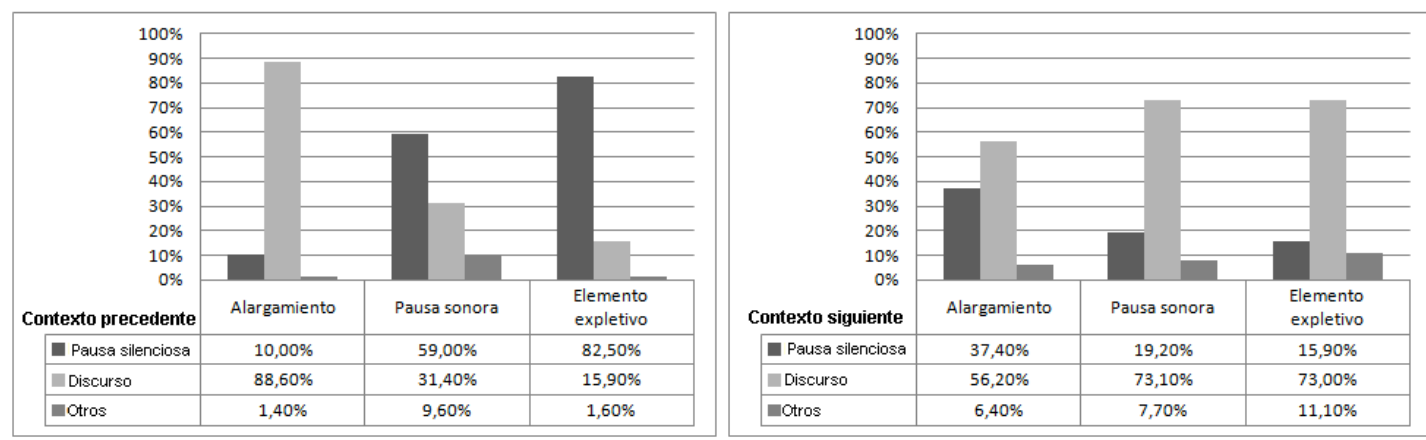

Figura 2. Frecuencia de aparición (en porcentajes) de los elementos que aparecen antes y después de las hesitaciones analizadas

La aparición de voz rota durante la emisión de las hesitaciones analizadas es otra característica más que se considera en este estudio. Como se puede apreciar en los datos que aparecen en la tabla 2 , en los que se muestran los casos de voz rota que se 
superponen a los fenómenos analizados, el porcentaje más alto se encuentra en los alargamientos, aunque, si consideramos los 500 casos analizados, solo un $21 \%$ del total presenta voz rota.

\begin{tabular}{|l|l|l|l|}
\hline & Pausas sonoras & Alargamientos & Elementos expletivos \\
\hline Sin voz rota & $82,1 \%$ & $75,8 \%$ & $84,1 \%$ \\
\hline Con voz rota & $17,9 \%$ & $24,2 \%$ & $15,9 \%$ \\
\hline
\end{tabular}

Tabla 2. Porcentajes de los casos de hesitación en los que aparece voz rota

La duración de las hesitaciones analizadas constituye otra de las variables que se han tenido en cuenta. Los resultados muestran que la duración no permite distinguir los alargamientos de las pausas sonoras ni de los elementos expletivos. Una prueba ANOVA de un factor en la que la variable dependiente es la duración y la independiente el tipo de fenómeno analizado así lo indica $(\mathrm{F}(2,498)=1.8$, n.s $)$. No obstante, como se puede apreciar en los valores de la tabla 3, el rango de duración es diferente en los tres fenómenos. El alargamiento puede llegar a una duración máxima de $2667 \mathrm{~ms}$. La pausa sonora es la que presenta el valor mínimo de duración más bajo, $126 \mathrm{~ms}$; mientras que el elemento expletivo muestra un valor mínimo de $228 \mathrm{~ms}$, el más alto si comparamos los tres fenómenos.

\begin{tabular}{|l|l|l|l|l|l|}
\hline & $\begin{array}{l}\text { Número de } \\
\text { casos }\end{array}$ & Valor medio & $\begin{array}{l}\text { Desviación } \\
\text { estándar }\end{array}$ & $\begin{array}{l}\text { Valor } \\
\text { mínimo }\end{array}$ & $\begin{array}{l}\text { Valor } \\
\text { máximo }\end{array}$ \\
\hline Pausa sonoras & 156 & 512,58 & 251,9 & 126 & 1292 \\
\hline Alargamientos & 281 & 478,66 & 233,96 & 177 & 2667 \\
\hline $\begin{array}{l}\text { Elementos } \\
\text { expletivos }\end{array}$ & 63 & 532,26 & 241,24 & 228 & 1610 \\
\hline
\end{tabular}

Tabla 3. Número de casos analizados, valores medios, desviaciones estándar, valores máximos y valores mínimos de duración (en ms) en las hesitaciones

Otra de las características que debemos considerar es qué tipo de pausa sonora es más habitual en español. Como se observa en la figura 3, en la que se puede apreciar qué vocalizaciones producen los hablantes, la más frecuente $(61,5 \%)$ es la que se emite como un sonido que se puede transcribir como [e:], representado como ehh en la figura, seguida de la que se pronuncia como un murmullo nasal (32\%), transcrita como $\mathrm{mmm}$; estas dos vocalizaciones constituyen el $93 \%$ de todos los casos analizados. Se muestran en la figura 3 muy pocos casos de vocalizaciones que se podrían transcribir como [a:] y unos pocos casos en que se combinan las dos vocalizaciones principales: el hablante empieza a realizar la pausa sonora como ehh y acaba con un murmullo nasal. 


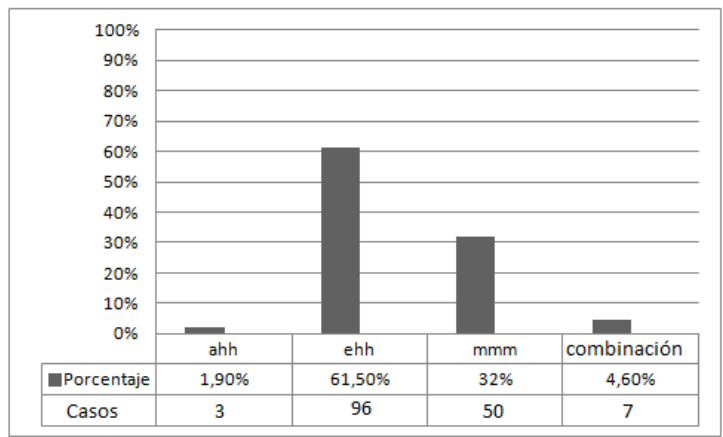

Figura 3. Tipos de vocalizaciones en la producción de una pausa sonora

Por último, se han determinado las categorías gramaticales de las palabras en las que se produce un alargamiento. Un $62 \%$ de los casos corresponden a palabras funcionales, mientras que un $38 \%$ a palabras con contenido léxico. La figura 4 muestra en qué palabras funcionales y en qué porcentaje se dan los alargamientos. Se ha creado una categoría específica «que», pues en la obtención de los datos se había observado que era una partícula en la que el hablante solía producir alargamientos independientemente de su categoría. No obstante, las partículas que presentan más alargamientos son las preposiciones, con un $40 \%$ de casos.

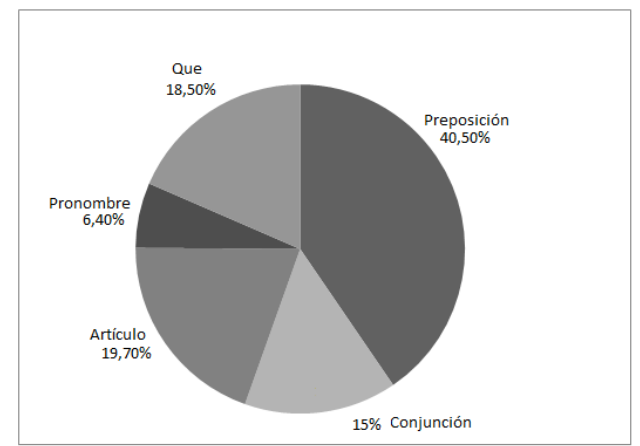

Figura 4. Porcentaje de casos de alargamiento considerando las diferentes categorías de palabras funcionales

\section{ESTUDIO PERCEPTIVO}

\subsection{Metodología}

Para el experimento de percepción se han seleccionado de las conversaciones seis casos representativos de la aparición de pausas sonoras y de alargamientos. Se han evitado aquellos enunciados en los que se daba una superposición de otros fenómenos (pausas silenciosas, voz rota, chasquidos o caídas en la frecuencia fundamental) que pudieran distorsionar la percepción de los elementos que se estaban evaluando. Una vez elegidos los enunciados, se han suprimido las pausas sonoras y los alargamientos para que los 
participantes en la prueba evaluaran la naturalidad y la espontaneidad de los mismos fragmentos con o sin alargamiento y con o sin pausa sonora. A modo de ejemplo, en el estímulo y cómo va a tener queeee convivir con ello pero bueno, se ha reducido la duración de la vocal alargada hasta obtener una duración similar a la de la vocal de la palabra que perteneciente a un enunciado en el que esta partícula aparecía sin alargamiento, de manera que se evaluaban dos fragmentos idénticos, uno con alargamiento y otro sin él. Lo mismo sucedía en la evaluación del papel de la pausa sonora; por ejemplo, en el enunciado pues habla en casa un castellano que tampoco es un castellano estándar ehhh sobre todo el castellano de mi casa se ha eliminado el elemento sonoro que duraba $156 \mathrm{~ms}$ y el sujeto percibía ambos fragmentos, con pausa sonora y sin ella. Por otra parte, para determinar si la duración de la pausa sonora influía en su percepción, en uno de los estímulos se duplicó la duración del elemento sonoro ehhh y se obtuvo un nuevo fragmento en el que la pausa sonora duraba $312 \mathrm{~ms}$. Para la manipulación de los estímulos se ha empleado el programa Praat (Boersma y Weenink, 2011).

En el test de percepción aparecían los estímulos de dos en dos de forma aleatoria (el fragmento natural y el manipulado) y se formulaban dos preguntas a los participantes. La primera tenía como objetivo evaluar la naturalidad y la espontaneidad de ambos fragmentos. En las instrucciones de la hoja de respuestas se definían los términos natural y espontáneo para que todos los sujetos partieran de la misma definición: «Natural quiere decir que los estímulos suenan a emisiones propias del español, mientras que espontáneo quiere decir que ese enunciado puede estar dentro de una conversación entre amigos que están tomando un café»». En la segunda pregunta se solicitaba a los participantes que indicaran si detectaban alguno de los siguientes fenómenos: alargamiento, pausa sonora, pausa silenciosa, o si no percibían ninguna diferencia entre los fragmentos que debían comparar. Debido a que la pausa sonora no es un concepto intuitivo para el hablante, se evitó el uso de este término en la hoja de respuestas, y se cambió por «un sonido que no pertenece a ninguna palabra dentro de la secuencia». La figura 5 muestra la hoja de respuestas con las preguntas que se formularon a los participantes. 
1. Escucha en el siguiente enlace dos realizaciones del estímulo pues habla en casa un castellano, que tampoco es un castellano estándar, sobre todo el castellano de mi casa... Contesta las siguientes preguntas:

a. ¿Cuál te parece más natural?

$\square$ La enunciación del fragmento 1 me parece más natural $\square$ La enunciación del fragmento 2 me parece más natural

$\square$ Suenan igual de naturales las dos enunciaciones

$\square$ No suenan naturales en español

b. ¿Cuál te parece más espontáneo?

\ La enunciación del fragmento 1 me parece más espontánea

\ La enunciación del fragmento 2 me parece más espontánea

$\square$ Suenan igual de espontáneas las dos enunciaciones

$\square$ No suenan espontáneas en español

c. Si tuvieras que indicar diferencias entre las dos pronunciaciones del estímulo, ¿qué diferencias marcarías?

$\square$ Hay un alargamiento de algún sonido en el primer fragmento que no está en el segundo

$\square$ Hay un alargamiento de algún sonido en el segundo fragmento que no está en el primero

$\square$ Aparece un sonido que no pertenece a ninguna palabra dentro de la secuencia en el primer fragmento, que no está en el segundo

$\square$ Aparece un sonido que no pertenece a ninguna palabra dentro de la secuencia en el segundo fragmento, que no está en el primero

$\square$ Hay una pausa en el primer fragmento que no está en el segundo

$\square$ Hay una pausa en el segundo fragmento que no está en el primero

$\square$ No puedo apreciar ninguna diferencia

Figura 5. Preguntas formuladas a los participantes en el test de percepción para evaluar las hesitaciones en los estímulos extraídos del corpus analizado 
En el experimento de percepción han participado 23 oyentes, 10 de ellos eran especialistas en fonética y los otros 13 eran estudiantes de segundo y de tercer año del Departamento de Filología Española de la Universidad Autónoma de Barcelona.

\subsection{Resultados}

En el análisis perceptivo no se muestra una diferencia significativa entre los oyentes expertos en fonética y los que poseen conocimientos básicos en esa materia. La prueba de chi2 así nos lo indica $(\mathrm{p}=.06)$. Por esta razón, el tratamiento estadístico de los datos se lleva a cabo de forma conjunta.

Los resultados que se presentan en la figura 6 muestran el modo en que los oyentes valoran la naturalidad de los estímulos en función de la presencia o la ausencia de alargamientos o de pausas. Los datos indican que los oyentes perciben como naturales tanto los estímulos en los que se compara la presencia con la ausencia de pausa sonora (un 68,5\%) como los estímulos en los que se compara la presencia con la ausencia del alargamiento (un 55,1\%). La diferencia entre los estímulos en los que se evalúa la pausa sonora y los estímulos en los que se evalúa el alargamiento no resulta significativa $(\mathrm{p}=.1)$.

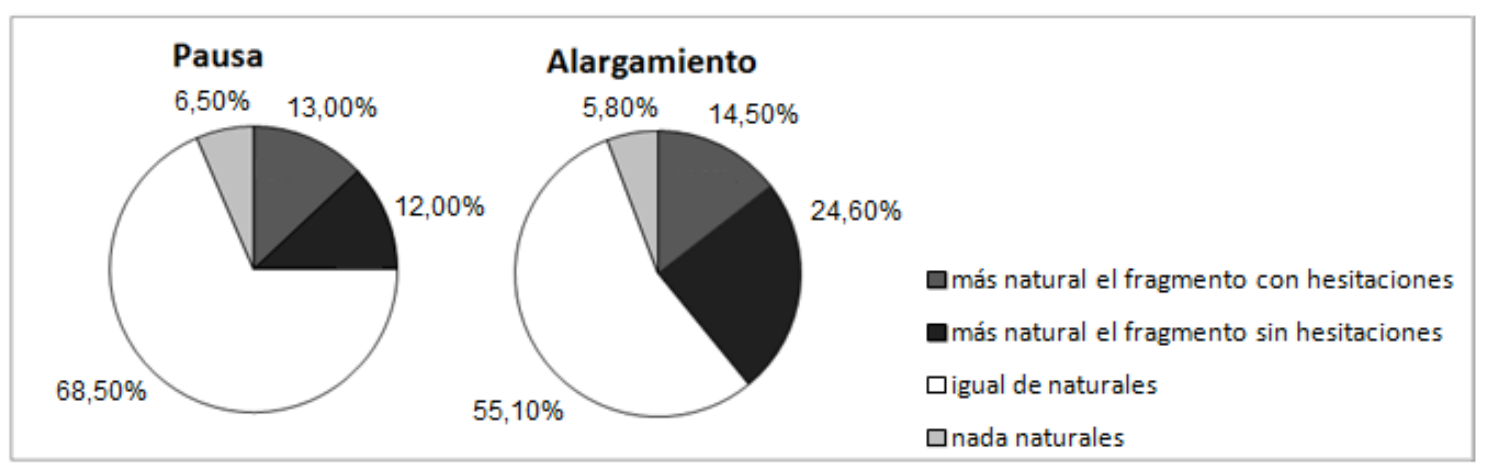

Figura 6. Valoración de la naturalidad de los estímulos en función de la presencia o de la ausencia de alargamientos o de pausas

Por otro lado, la percepción de la espontaneidad varía de forma significativa $(\mathrm{p}=.000)$ cuando se contrastan los resultados de las pausas sonoras y los de los alargamientos (véase la figura 7). Los estímulos en los que se compara la presencia o la ausencia de la pausa sonora se perciben con el mismo grado de espontaneidad (42,4 \%). Por el contrario, si se observan los resultados de los estímulos con alargamiento frente a aquellos en los que se ha elidido, un $66,7 \%$ de las respuestas indican que se percibe como más espontáneo el fragmento en el que aparece el alargamiento. 


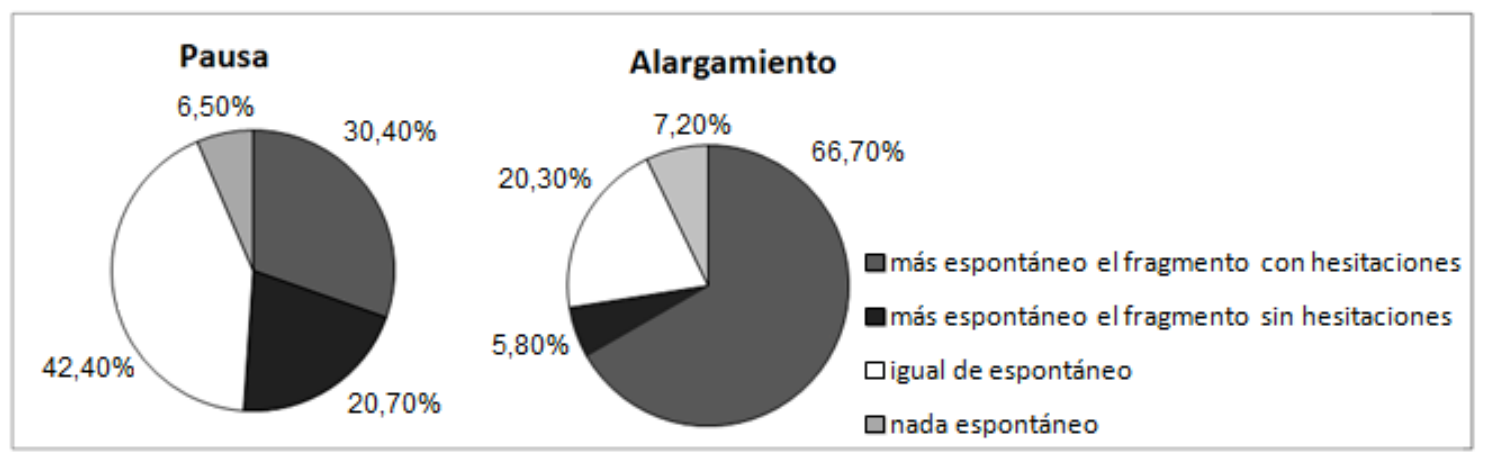

Figura 7. Valoración de la espontaneidad de los estímulos en función de la presencia o de la ausencia de alargamientos o de pausas

Por último, cabe observar qué perciben los oyentes en cada uno de los seis estímulos incluidos en la prueba cuando no identificaban el fenómeno que se presentaba ( $29 \%$ para los casos de pausas y $23 \%$ para los de alargamientos). La figura 8 muestra para cada estímulo las respuestas de los oyentes. En algunos estímulos con pausas sonoras identificaban alargamientos (estímulo 3) y en algunos casos de alargamientos percibían pausas sonoras (estímulo 5). Llama la atención el hecho de que se perciban como iguales $(81,30 \%)$ dos fragmentos que se diferenciaban por la presencia o la ausencia de una pausa sonora de 152 ms (estímulo 2), y también que la mayoría de los casos en los que no se identifica el fenómeno presentado coincidan con aquellos en los que se percibe una pausa silenciosa que no aparecía en los estímulos, sobre todo en los que se corresponden con un alargamiento (estímulos 1,4 y 5).

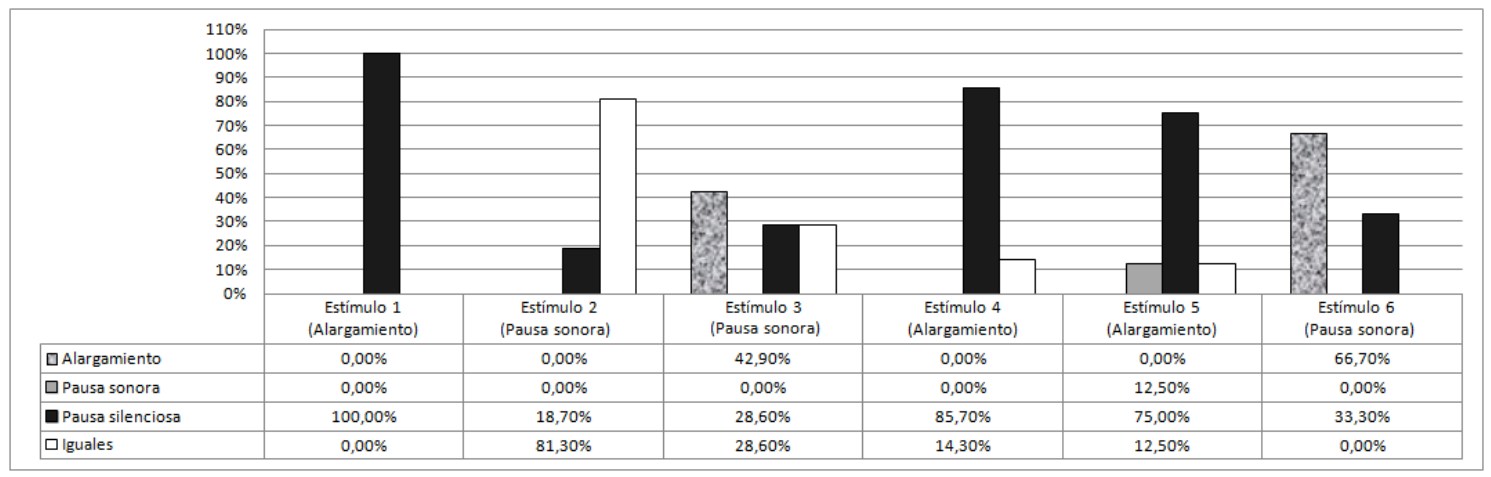

Figura 8. Fenómenos percibidos en los casos en los que los oyentes no identifican el fenómeno presentado en el estímulo. Para cada estímulo se indica entre paréntesis el fenómeno evaluado

\section{CONCLUSIONES}

Los resultados muestran que, aunque las pausas sonoras y los alargamientos suponen una interrupción en el discurso del hablante, la aparición estas hesitaciones afecta relativamente a la efectividad de la comunicación, como lo demuestra el hecho de que en el experimento de percepción se han identificado los dos tipos de estímulos 
presentados -con pausas sonoras/sin pausas sonoras o con alargamientos $/$ sin alargamientos- como naturales.

A pesar de que algunos autores señalan que estos fenómenos se utilizan en el discurso para indicar a los oyentes cuál es el momento más propicio en la conversación para tomar el turno de palabra (véase, por ejemplo, Cesteros, 2000), se pueden encontrar los mismos elementos en aquellas situaciones comunicativas en las que el hablante sabe que no va a ser interrumpido por su interlocutor; en estos casos, incluso puede llegar a incrementarse la frecuencia de aparición de las hesitaciones.

Por otro lado, las pausas sonoras y los expletivos suelen estar precedidos de otros elementos que interrumpen el discurso, como las pausas silenciosas, mientras que el alargamiento, generalmente, viene precedido de habla, ya que también puede producirse en la vocal inicial de la palabra. No obstante, si consideramos los elementos que aparecen después de las hesitaciones, los alargamientos seguidos de pausa silenciosa son los más frecuentes. Además, la mayoría de los casos en los que se produce voz rota se corresponden con un alargamiento.

Se ha observado también que los valores medios y las desviaciones de la duración no permiten distinguir estos fenómenos, pero sí es importante considerar los valores mínimos y los máximos. Los alargamientos presentan los valores máximos de duración, mientras que las pausas sonoras presentan los valores mínimos.

Cabría analizar con más detalle qué duración mínima debe tener una pausa sonora para que no pase inadvertida al oyente. No se ha de olvidar que en el experimento de percepción una pausa sonora de $156 \mathrm{~ms}$ no se ha percibido, pues los oyentes han identificado los dos fragmentos, el que contenía pausa sonora y el que carecía de ella, como iguales.

El análisis muestra que la vocalización más frecuente en español para la pausa sonora es un elemento vocálico que se puede representar como [e:], resultado que coincide con los de otros trabajos (Rebollo, 1997). No obstante, en este estudio no se han analizado los valores de los formantes de la pausa sonora para poder afirmar que realmente su estructura formántica es la correspondiente a una vocal media palatal en español. En cuanto a la categoría léxica en la que se ha producido un alargamiento, la preposición es la que presenta el mayor porcentaje de casos.

Finalmente, los resultados del experimento de percepción muestran que los alargamientos se perciben más frecuentemente como pausas silenciosas que como pausas sonoras. Aun así, los oyentes, en ocasiones, identifican ambas hesitaciones como silencios, aunque en ninguno de los estímulos apareciera una pausa silenciosa; quizá los datos obtenidos sugieren que estos fenómenos podrían marcar en el discurso un límite prosódico, ejerciendo la misma función que los silencios. Además, cabe mencionar que los oyentes no advierten una vocalización de $156 \mathrm{~ms}$ de duración en medio del enunciado, quizá porque la comunicación sigue siendo efectiva. Se necesitaría un estudio exhaustivo que permitiera determinar qué duración han de tener estas hesitaciones para que sean percibidas por el oyente. 


\section{REFERENCIAS BIBLIOGRÁFICAS}

Albalá, M. J., E. Battaner, M. Carranza, C. De la Mota, J. Gil, J. Llisterri y A. Ríos (2008): «VILE: Análisis estadístico de los parámetros relacionados con el grupo de entonación», en Pamies, A., M. C. Amorós y J. M. Pazos, eds., Language Design. Journal of Theoretical and Experimental Linguistics. Special Issue 2: Experimental Prosody, Granada, Método Ediciones, 15-22.

Boersma, P. y D. Weenink (2011): Praat: Doing phonetics by computer (versión 5.3.02): [en línea]: <http://www.praat.org/>.

BRANDER, D. (2014): «Phonetic characteristics of hesitation vowels in Swiss German and their use for forensic speaker identificacion», comunicación presentada en IAFPA 2014. 23rd Annual Conference of the International Association for Forensic Phonetics and Acoustics, Zúrich, Suiza. [en línea]: http://www.pholab.uzh.ch/static/IAFPA/abstracts/BRANDERdario.pdf

CAlsamiglia, H. y A. Tusón (2007): Las cosas del decir. Manual de análisis del discurso (2. ${ }^{a}$ edición actualizada), Barcelona, Ariel.

Candeias, S., D. Celorico, J. ProençA, A. Veiga y F. Perdigão (2013): «HESITA(tions) in Portuguese: a database», en R. Eklund, ed., Proceedings of DiSS 2013. 6th Workshop on Disfluency in Spontaneous Speech, Estocolmo, 1316.

Carlson, R., K. Gustafson y E. Strangert (2006): «Prosodic cues for hesitation», Lund Working Papers in Linguistics, vol. 52, 21-24.

Cestero, A. M. (2000): El intercambio de turnos de habla en la conversación. Análisis sociolingüístico, Alcalá de Henares, Universidad de Alcalá de Henares, Servicio de Publicaciones.

CiCRES, J. (2007): «Análisis discriminante de un conjunto de parámetros fonéticoacústicos de las pausas llenas para identificar hablantes», Sintesis Tecnológica, 3(2), 87-96, <http://dx.doi.org/10.4206/sint.tecnol.2007.v3n2-04>.

CICRES, J. (2014): «Comparación forense de voces mediante el análisis multidimensional de las pausas llenas», Revista Signos. Estudios de Lingüística, 47(86), 365-384, <http://dx.doi.org/10.4067/s0718-09342014000300002>.

CORTÉs, L. (1991): Sobre conectores, expletivos y muletillas en el español hablado, Málaga, Ágora.

Crystal, D. (2008): A dictionary of linguistics and phonetics (6. ${ }^{\mathrm{a}}$ edición), Oxford, Blackwell.

Gayraud, F., H.-R. LeE y M. Barkat-Defradas (2011): «Syntactic and lexical context of pauses and hesitations in the discourse of Alzheimer patients and healthy elderly subjects», Clinical Linguistics y Phonetics, 25(3), 198-209, $<$ http://dx.doi.org/10.3109/02699206.2010.521612>.

GIL, J. (2007): Fonética para profesores de español: de la teoría a la práctica, Madrid, Arco/Libros.

KÜNZEL, H. J. (1997): «Some general phonetic and forensic aspects of speaking tempo», International Journal of Speech, Language and the Law, 4(1), 48-83. 
Nicholson, H., Eberhard, K. y Scheutz, M. (2010): «“Um... I don’t see any”: The function of filled pauses and repairs», en Proceedings of DiSS-LPSS Joint Workshop 2010. 5th Workshop on Disfluency in Spontaneous Speech and 2nd International Symposium on Linguistic Patterns in Spontaneous Speech, Tokio, 89-92.

OrtegA, J., J. GonZÁLEZ y V. MARRERo (2000): «AHUMADA: A large speech corpus in Spanish for speaker characterization and identification», Speech Communication, $\quad 31(2-3), \quad 255-264, \quad<$ http://dx.doi.org/10.1016/s01676393(99)00081-3>.

Real ACAdemia Española (2014): Diccionario de la lengua española (23. a edición), Madrid, Espasa Calpe.

Rebollo, L. (1997): «Pausas y ritmo en la lengua oral. Didáctica de la pronunciación», en Moreno, F., M. Gil y K. Alonso (eds.), El español como lengua extranjera: del pasado al futuro. Actas del VIII Congreso Internacional de la Asociación para la Enseñanza del Español como Lengua Extranjera, Alcalá de Henares, Servicio de Publicaciones de la Universidad de Alcalá, 667-676.

RodríGUEZ, J. (2013): "Las pausas en el discurso de individuos con demencia tipo Alzheimer. Estudio de casos», Lengua y Habla, vol. 17, 253-267.

Rodríguez, J. L., M. I. TORRES y A. VARONA (2001): «Annotation and analysis of disfluencies in a spontaneous speech corpus in Spanish», en Proceedings of DiSS 2001. ISCA Tutorial and Research Workshop (ITRW) on Disfluency in Spontaneous Speech, Edimburgo, 1-4.

Rose, R. (1998): The communicative value of filled pauses in spontaneous speech, tesis doctoral, Birmingham, University of Birmingham [en línea]:

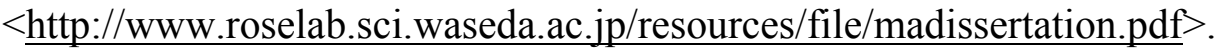

Rose, R. (2013): "Crosslinguistic Corpus of Hesitation Phenomena: A corpus for investigating first and second language speech performance», en Proceedings of Interspeech 2013. 14th Annual Conference of the International Speech Communication Association, Lión, 992-996.

SÁNCHEZ, C. (2002): «La percepción de la fluidez en español como segunda lengua», Revista de Filología y Lingüística de la Universidad de Costa Rica, 28(1), 137163.

Stenström, A.-B. (1994): An introduction to spoken interaction, Londres, Longman.

STEPANOVA, S. (2007): "Some features of filled hesitation pauses in spontaneous Russian», en Trouvain, J. y W. J. Barry, eds., Proceedings of ICPhS 2007. 16th International Congress of Phonetic Sciences, Saarbrücken, 1325-1328.

TORRES, M. I. (2006): «El reconocimiento del habla», en Llisterri J. y M. J. Machuca (eds.), Los sistemas de diálogo, Bellaterra-Soria: Universitat Autònoma de Barcelona-Fundación Duques de Soria, 81-98.

WenNerstrom, A. (1994): «Intonational meaning in English discourse: A study of non-native speakers», Applied Linguistics, 15(4), 399-420, $<\underline{\text { http://dx.doi.org/10.1093/applin/15.4.399> }}$. 